\title{
Online K-Means Algorithm for Background Subtraction
}

\author{
Peng Chen ${ }^{1,}$, Beibei Jin $^{1}$, Xiangbing Zhu ${ }^{1}$, Mingxing Fang ${ }^{1}$ \\ ${ }^{1}$ College of Physics and Electronic Information, Anhui Normal University, WuHu 241000, China, \\ aemail: ahnuchp@mail.ahnu.edu.cn
}

Keywords: Gaussian mixture model; on-line K-means; background subtraction

\begin{abstract}
Background subtraction is an important step in video processing. GMM algorithm uses Gaussian mixture model to identify moving objects and efficient equations have been derived to update GMM parameters. In order to compute parameters more accurately while maintain constant computing time per frame, we apply online K-Means algorithm to update the parameters of Gaussian mixture models and the corresponding incremental K-means equations are derived. Experiments demonstrate that online K-means algorithm can give more efficient segment result than previous update equations.
\end{abstract}

\section{Introduction}

Background subtraction is to separate foreground pixels from background pixels in a video. Parametric background modeling methods detect all the moving objects (foreground) in a video stream by building a representation of the scene called the background model. Over the years, numerous algorithms have been proposed for background subtraction such as Gaussian Mixture Model (GMM)[1] and the Kernel Density Estimator(KDE) [2]. In the case of pixel-level background model, the background can be described by a probability density function (PDF) for each pixel separately. Single Gaussian model is one of such models and was proposed by Wren et al.[3]. Although single Gaussian model has been used successfully for indoor scenes, for more complex outdoor scenes, it is not a good model [4] and elaborate models are needed. A substantial improvement in background modeling is achieved by using multimodal models such as GMM to describe per-pixel background color. In GMM, each pixel value is modeled as a mixture of Gaussians. A pixel from a new image is considered to be a background pixel if its new value is well described by its background density function. Otherwise, the pixel is classified as foreground. Efficient update equations in GMM are given in [1] with a fixed number of Gaussian components. Zoran and Ferdinand proposed an adaptive method which can automatically select the right number of components [5]. On-line Expectation Maximization (EM) algorithm has been used to estimate the GMM parameters [6]. In this paper, we describe the GMM method in K-Means framework and show that the foreground objects can be detected more efficiently if the parameters of GMM are calculated by online K-means method.

The paper is organized as follows. In the next section, we review GMM background subtraction approach. In Section 3, online K-means algorithms are introduced. Experimental results for a simple Gaussian mixture models and video based background subtraction are presented in Section 4 before conclusion in Section 5.

\section{Gaussian Mixture Model}

For outdoor scenes, multiple colors can be observed at a certain location due to complicated outdoor scenes and illumination variations. In this case, multinomial PDFs such as GMM have been introduced to model the background. In more detail, GMM models each pixel with a mixture of $\mathrm{M}$ Gaussians. Thus, at time t, the probability of occurrence of a pixel value is represented as

$$
p\left(x_{t} \mid \theta\right)=\sum_{i=1}^{M} w_{i} N\left(x_{t} \mid \mu_{i}, \Sigma_{i}\right)
$$


Where $\mathrm{w}_{\mathrm{i}} \geq 0$ and $\sum \mathrm{w}_{\mathrm{i}}=1$. The parameter vector $\theta$ consists of the mixing proportions wi, the mean vectors $\mu_{\mathrm{i}}$ and the covariance matrices $\sum_{\mathrm{i}}$.

For a given pixel location, we assume that its $\mathrm{N}$ pixel values $\mathrm{X}=\{\mathrm{x} 1, \mathrm{x} 2, \mathrm{x} 3 \cdots \mathrm{xN}\}$ is independent identically distributed. We can obtain estimations of $\theta$ by maximizing log likelihood function with standard EM algorithm [6]. In this paper we use K-Means algorithm to estimation $\theta$ more efficiently.

For every pixel location, we use K-Means algorithm to determine the corresponding GMM parameter vectors. To classify the new pixel, we use the following rule [5]:

$$
\left\{p(x \mid B G)=\sum_{i=1}^{B} w_{i} N_{i}\left(x \mid \mu_{i}, \Sigma_{i}\right)\right\}>c_{t h r}
$$

Where cthris a constant threshold. If (2) is satisfied, the new pixel should be classified as a background pixel. The components are sorted in descending order by its proportions $\mathrm{w}_{\mathrm{i}} \cdot \mathrm{p}(\mathrm{x} \mid \mathrm{BG})$ is the background model which is represented by $\mathrm{B}$ largest Gaussian components of all $\mathrm{M}$ components in (1). B can be determined by

$$
B=\underset{b}{\arg \min }\left(\sum_{j=1}^{b} w_{j}>c_{b}\right)
$$

Where $c_{b}$ is a measure of the minimum portion of the pixels that can approximate background. The attractiveness of the K-Means algorithm is that it is easy to implement and can rapidly converge to a local maximum of the log-likelihood function. However, this algorithm needs to collect all the image frames to compute the GMM parameters, which demands huge amount of memory and makes it unsuitable to be used in real time applications. To overcome this difficulty while maintaining the advantages of K-Means algorithm, we bring forth an online K-Means approach in the next section.

\section{Online K-Means Algorithm}

As the online K-Means algorithm is derived from the online EM algorithm, we will introduce the online EM algorithm and online K-Means algorithm respectively.

a)Sufficient Statistics Based Online EM Algorithms

The first online algorithm studied in this paper is the one presented by [7], which is based on sufficient statistics.

online EM algorithm:

\section{1: $\quad$ E-step}

Calculate the posterior probability $\varepsilon_{i}^{\mathrm{t}}$ that i-th component is responsible for generating $\mathrm{xt}$ [6].After each new data arrived, update sufficient statistics vectors si for each component:

$$
s_{i}^{\text {new }}=<\sum_{j=1}^{t} \varepsilon_{i}^{j}, \sum_{j=1}^{t} \varepsilon_{i}^{j} x_{j}, \sum_{j=1}^{t} \varepsilon_{i}^{j} x_{j} x_{j}^{T}>=s_{i}^{\text {old }}+<\varepsilon_{i}^{j}, \varepsilon_{i}^{j} x_{j}, \varepsilon_{i}^{j} x_{j} x_{j}^{T}>, i=1 \ldots M
$$

\section{M-step}

Maximize the likelihood function with respect to parameter $\theta$ :

$$
\begin{aligned}
& w_{i}=s_{i 1} /\left(s_{11}+s_{21}+\cdots+s_{M 1}\right) \\
& \mu_{i}=s_{i 2} / s_{i 1} \\
& \Sigma_{i}=s_{i 3} / s_{i 1}-s_{i 2} s_{i 2}^{T} /\left(s_{i 1} s_{i 1}\right) \\
& s_{i 1}=\sum_{j=1}^{t} \varepsilon_{i}^{j}, s_{i 2}=\sum_{j=1}^{t} \varepsilon_{i}^{j} x_{j}, s_{i 3}=\sum_{j=1}^{t} \varepsilon_{i}^{j} x_{j} x_{j}^{T}
\end{aligned}
$$

Where $s_{i 1}, s_{i 2}$ and $s_{i 3}$ are the corresponding element of sufficient statistics vectors for the i-th component respectively. 


\section{b)Sufficient Statistics Based Online K-Means Algorithms}

Due to the fact that K-Means algorithm can be seen as a special case of EM algorithm if the variance tends to zero, we can derive an online K-Means algorithm as follows:

online K-Means:

1: initialize parameters $w_{i}, \mu_{i}, \Sigma_{i}, \mathrm{i}=1, \ldots \mathrm{K}$

2: E step: Assign new data to the nearest component which is responsible for generating xt.After each new data arrived, update sufficient statistics vectors si for each component:

$$
s_{k}^{\text {new }}=<\sum_{j=1}^{t} r_{k}^{j}, \sum_{j=1}^{t} r_{k}^{j} x_{j}, \sum_{j=1}^{t} r_{k}^{j} x_{j} x_{j}^{T}>=s_{k}^{\text {old }}+<\mathrm{r}_{k}^{t}, \mathrm{r}_{k}^{t} x_{t}, \mathrm{r}_{k}^{t} x_{t}^{T} x_{t}>, k=1 \ldots K
$$

where $r_{k}{ }^{j}$ is 0 or 1 . if $x_{j}$ is nearest to $\mu_{k}$, then $r_{k}{ }^{j}$ is 1 , otherwise $r_{k}^{j}$ is 0 .

3: M step: update parameters:

$$
\begin{aligned}
& w_{i}=s_{i 1} /\left(s_{11}+s_{21}+\cdots+s_{K 1}\right) \\
& \mu_{i}=s_{i 2} / s_{i 1} \\
& \sum_{i}=s_{i 3} / s_{i 1}-s_{i 2} s_{i 2}^{T} /\left(s_{i 1} s_{i 1}\right)
\end{aligned}
$$

Where $s_{i 1}, s_{i 2}$ and $s_{i 3}$ are the corresponding element of sufficient statistics vectors for the i-th component respectively.

As $\sum_{\mathrm{i}}$ is a full 3 by 3 covariance matrix, we need to computer its inverse and determinant in $\mathrm{E}$ step. In order to simplify calculation, we can keep covariance matrices isotropic by making $\sum_{\mathrm{i}}$ equal to $\sigma_{\mathrm{i}}{ }^{2} * \mathrm{I}_{3 \times 3}$. Now the parameter vector $\theta$ consists of the mixing proportions wi, the mean vectors $\mu_{\mathrm{i}}$ and the covariance value $\sigma_{i}{ }^{2}$. By maximizing log-likelihood, we can derive similar iterative equations for isotropic covariance matrices based online K-Means algorithm.

c)simplified online K-Means:

1: initialize parameters $w_{i}, \mu_{i}, \Sigma_{i}, \mathrm{i}=1, \ldots \mathrm{K}$

2: E step: Assign new data to the nearest component which is responsible for generating $\mathrm{x}_{\mathrm{t}}$. After each new data arrived, update sufficient statistics vectors $\mathrm{s}_{\mathrm{i}}$ for each component:

$$
s_{k}^{\text {new }}=s_{k}^{\text {old }}+<\mathrm{r}_{k}^{t}, \mathrm{r}_{k}^{t} X_{t}, \mathrm{r}_{k}^{t} X_{t}^{T} X_{t}>, k=1 \ldots K
$$

3: M step: update parameters:

$$
\begin{aligned}
& w_{i}=s_{i 1} /\left(s_{11}+s_{21}+\cdots+s_{K 1}\right) \\
& \mu_{i}=s_{i 2} / s_{i 1} \\
& \sum_{i}=\left(s_{i 3} / s_{i 1}-s_{i 2}^{T} s_{i 2} /\left(s_{i 1} s_{i 1}\right)\right) / 3
\end{aligned}
$$

Where $s_{11}, s_{i 2}$ and $s_{i 3}$ are the corresponding element of sufficient statistics vectors for the i-th component respectively.

\section{Test results}

a)Mixture model result

In order to demonstrate that the online K-Means algorithm can find the correct parameters of GMM model, we have applied it to a 3 component 2 dimensional GMM model. We synthetically generated data points from this GMM model and then applied the standard EM and the online K-Means to estimate the model's parameters. For standard EM, we use 1000 data items as input and 
iterate EM steps 100 times. As each data item will be visited only once in online K-Means, we use 100000 data items for it to make online K-Means and standard EM go through the same number of EM steps. Fig 1 shows the results. We can see from Fig 1 that the online K-Means is a good approximation to the standard EM. If the covariance is not important, then the simplified online K-Means with isotropic covariance matrices can also be considered.

b)Background subtraction result

To demonstrate the effectiveness of the proposed method for background subtraction, we compare online K-Means based background subtraction results with that of Adaptive Density Estimate (ADE) [5]. Experimental results reveal that our method can reduce the noise around segmented foreground objects (Fig 2).

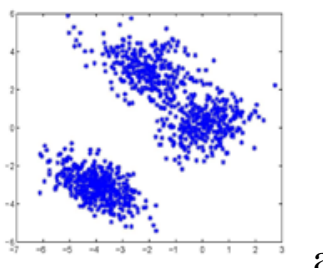

a

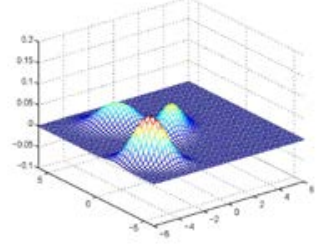

b

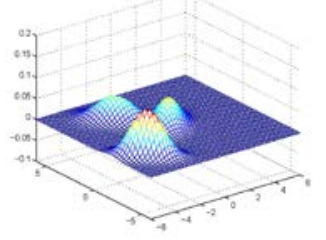

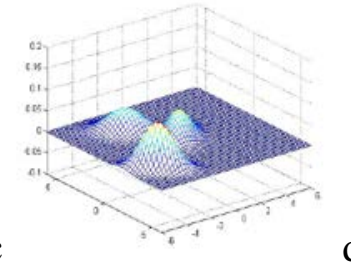

Fig. 1. GMM models result. (a) Data generated. (b)True PDF. (c) Standard EM (d) online Kmeans
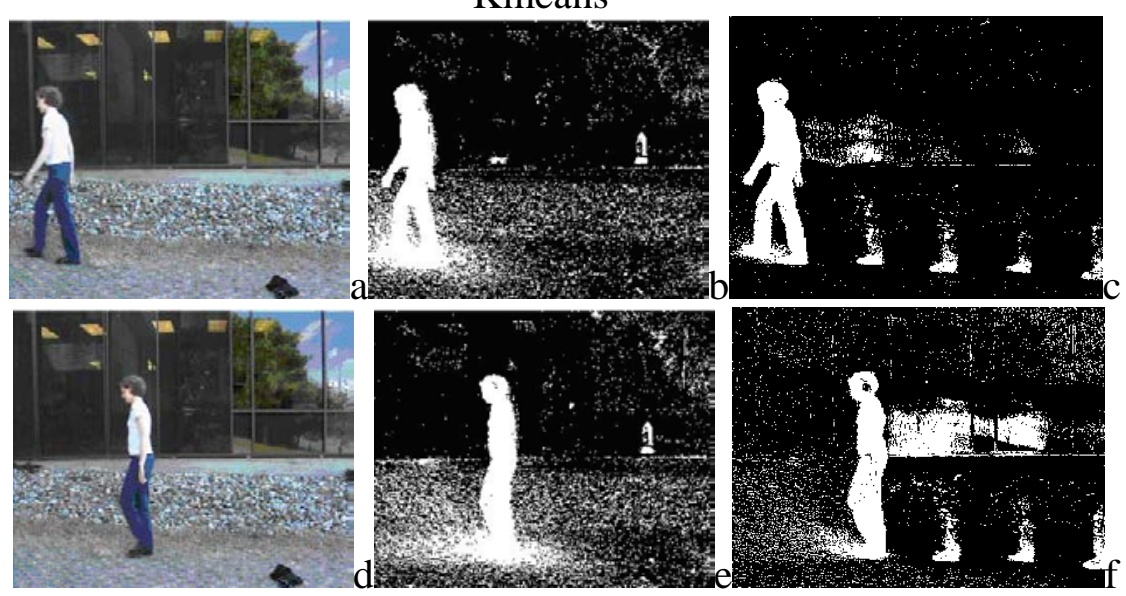

Fig. 2. Background subtraction results. (a) frame 96; (b) ADE for Frame 96; (c) Online KMeans for Frame 96;(d)frame 76;(e) ADE for Frame 76;(f)online KMeans for Frame 76.

\section{Conclusion}

Given background subtraction in a broad range of applications, adoption of the new technique provides significant benefits in a number of areas. In this paper, we propose a novel approach by using online K-Means algorithm. Our method can recognize foreground objects more accurately compared to the ADE. Future research includes exploring cohesiveness of adjacent pixels instead of relying solely on each pixel in isolation. Also, how to exploit semantics of objects from high level modules to feedback the detection is needed to focus on. A more robust model for dynamic background is another issue.

\section{Acknowledgement}

In this paper, the research was supported by Natural Science Foundation of Anhui Province, China (No.090414173), The Innovation Funds of Anhui Normal University.

\section{References}

[1] C. Stauffer and W.E.L. Grimson, “Adaptive background mixture models for real-time tracking," in IEEE Computer Society Conference on Computer Vision and Pattern Recognition., 1999, vol. 2, pp. 246-252. 
[2] Ahmed Elgammal, David Harwood, and Larry Davis, "Non-parametric model for background subtraction,” in Computer Vision-ECCV 2000, pp. 751-767. 2000.

[3] Christopher Richard Wren, Ali Azarbayejani, Trevor Darrell, and Alex Pentland, "Pfinder: Real-time tracking of the human body," IEEE Transactions on Pattern Analysis and Machine Intelligence, vol. 19, no. 7, pp. 780- 785, 1997.

[4] X. Gao, T. E. Boult, F. Coetzee, and V. Ramesh, "Error analysis of background adaption,” in CVPR, 2000, pp. I: 503-510.

[5] Zoran Zivkovic and Ferdinand van der Heijden, "Efficient adaptive density estimation per image pixel for the task of background subtraction,” Pattern Recognition Letters, vol. 27, no. 7, pp. 773 780, 2006.

[6] Bishop C M. Pattern Recognition and Machine Learning[M]. 1st ed. Cambridge:Springer, 2008.

[7] R. M. Neal and G. E. Hinton, “A new view of the EM algorithm that justifies incremental, sparse and other variants,” in Learning in Graphical Models, M. I. Jordan, Ed., pp. 355-368. Kluwer Academic Publishers, 1998.

[8] S. J. Nowlan, Soft Competitive Adaptation: Neural Network Learning Algorithms based on Fitting Statistical Mixtures, Ph.D. thesis, School of Computer Science, Carnegie Mellon University, Pittsburgh, PA, Apr. 1991. 